\title{
A Reversible Runtime Environment for Parallel Programs
}

\author{
Takashi Ikeda and Shoji Yuen ${ }^{(\varpi)}$ \\ Graduate School of Informatics, Nagoya University, \\ Furo-cho, Chikusa-ku, Nagoya 464-8601, Japan \\ \{tikeda, yuen\}@sqlab.jp
}

\begin{abstract}
We present a reversible runtime environment for simple parallel programs and its experimental implementation. We aim at a lightweight implementation of the backtrack reversibility by the state-saving mechanism using stacks. We translate a program to a sequence of simple commands of an executable intermediate representation for reversible stack machines. The parallel composition is implemented using the multiprocessing feature of Python. While executing the commands, the stack machines collect the information for the backward execution in the auxiliary stacks for the update history of the variables and the history of jumps. The commands for the backward execution is obtained by reversing the commands for the forward execution by replacing each command with the corresponding reversed command. In the purpose of behaviour analysis with reversibility such as debugging, our runtime is more portable than the source-to-source translation of a high-level programming language.
\end{abstract}

Keywords: Reversible computation · Imparative parallel programs • Stack machine $\cdot$ Python multiprocessing

\section{Introduction}

Reverse execution of programs has been investigated based on the reversible computing recently. Undoing the effect of an execution of a program till returning to the initial state is useful in analysing the finer-grained behavioural property of the program. In general, the execution of a parallel program depends on the environment such as the scheduler and I/O channels. Replaying the program may not reach the same states as the previous run. This makes behavioural analysis difficult to work out the cause of the defect for debugging.

Reversible programming languages such as Janus [4,7] and RFUN [6] are designed for the reversed execution at the level of the design of programming languages. For example, Janus needs the extra-control structure at the end of the conditional branch in order to know which branch is executed to reverse the conditional statement. For this approach, the state-saving mechanism is not needed 
since the computation is fully reversed. However, introducing parallel composition becomes difficult since the runtime environment is not directly described in programs.

We present a reverse execution mechanism that the runtime collects the information in stacks at a forward execution. At the reverse execution, the runtime executes the program simply in the reversed order using the information stored in the stacks. A source program is compiled to a sequence of simple commands executed by the stack machine. Each process in the parallel composition is dispatched to each stack machine forked from the initial stack machine. We implement the backtrack reversibility with the multiprocessing feature of Python. In the reverse execution, multiple stack machines are invoked, but they are controlled by the stacks to follow back the forward execution.

The report is structured as follows. Section 2 gives the syntax of the programs, Sect. 3 presents the stack machine design and Sect. 4 states the concluding remarks.

\section{Programming Language with Parallel Composition}

Our parallel programming language is defined as follows where ()$^{+}$and ()$^{*}$ denotes the repetition of one or more times and zero or more times respectively:

$$
\begin{aligned}
& P::=D Q R \mid D Q \text { par }\{Q\}(\{Q\})^{+} R \\
& D::=(\operatorname{var} X ;)^{*} \\
& R::=(\text { remove } X ;)^{*} \\
& Q::=(S ;)^{*} S \\
& S::=\operatorname{skip}|X=E| \text { if } C \text { then } Q \text { else } Q \text { fi } \mid \text { while } C \text { do } Q \text { od } \\
& E::=X|n| E \text { op } E \mid(E) \\
& C::=B|C \& \& C| \operatorname{not} C \mid(C) \\
& B::=E=E \mid E<E
\end{aligned}
$$

The language is a simplified version of that in $[2,3]$. par denotes the parallel composition of sequential procedures. For simplicity, we remove the nested block structure and procedures. remove statements at the end of a program correspond to the variables declared at the beginning of the program, where the order of declarations is supposed to be reversed. For example, if variables are declared as var $X$; var $Y$, the variables are removed as remove $Y$; remove $X$. This ensures the correspondence between the variable and the entry of the symbol table.

\section{Reversible Execution of Stack Machine Code}

\subsection{Reversible Stack Machine}

For simplicity, a parallel program in this report is limited in the form that an initial stack machine runs first followed by parallel blocks. For values, we consider 
the integers Z. $\mathbb{A}$ is the set of address $\mathbb{A}$ as the locations of commands in a stack machine code. Here an address is a positive natural number. $\mathbb{P}$ is the set of stack machine identifiers (SMid). An SMid is a natural number. We assume the initial stack machine has the SMid of 0 . Other stack machines have id's in a row from 1 to $N$ where $N$ is the number of parallel blocks.

The stack machine configuration is $\left(P C, P C^{\prime}, w, \rho, \xi\right)_{\sigma}$ where $P C$ is the program counter, $P C^{\prime}$ holds the previous $\mathrm{PC}$ value, $w \in \mathbb{Z}^{*}$ is a local stack, $\rho \in(\mathbb{A} \times \mathbb{P})^{*}$ is a label stack, and $\xi \in(\mathbb{Z} \times \mathbb{P})^{*}$ is a value stack. $\sigma$ is a symbol table that maps a variable to its value. $\sigma(v)$ presents the value of $v$. For the local stack $w$ and $z \in \mathbb{Z}, z w$ is the concatenation of $z$ and $w$.

Each stack machine is identified by $(p, N)$ with $p$ is a process identifier and $N$ is a number of all parallel blocks in a program. The behaviour of a stack machine $S M_{(p, N)}$ for command $c$ is specified by $\stackrel{c}{\rightarrow}(p, N)$ as follows:

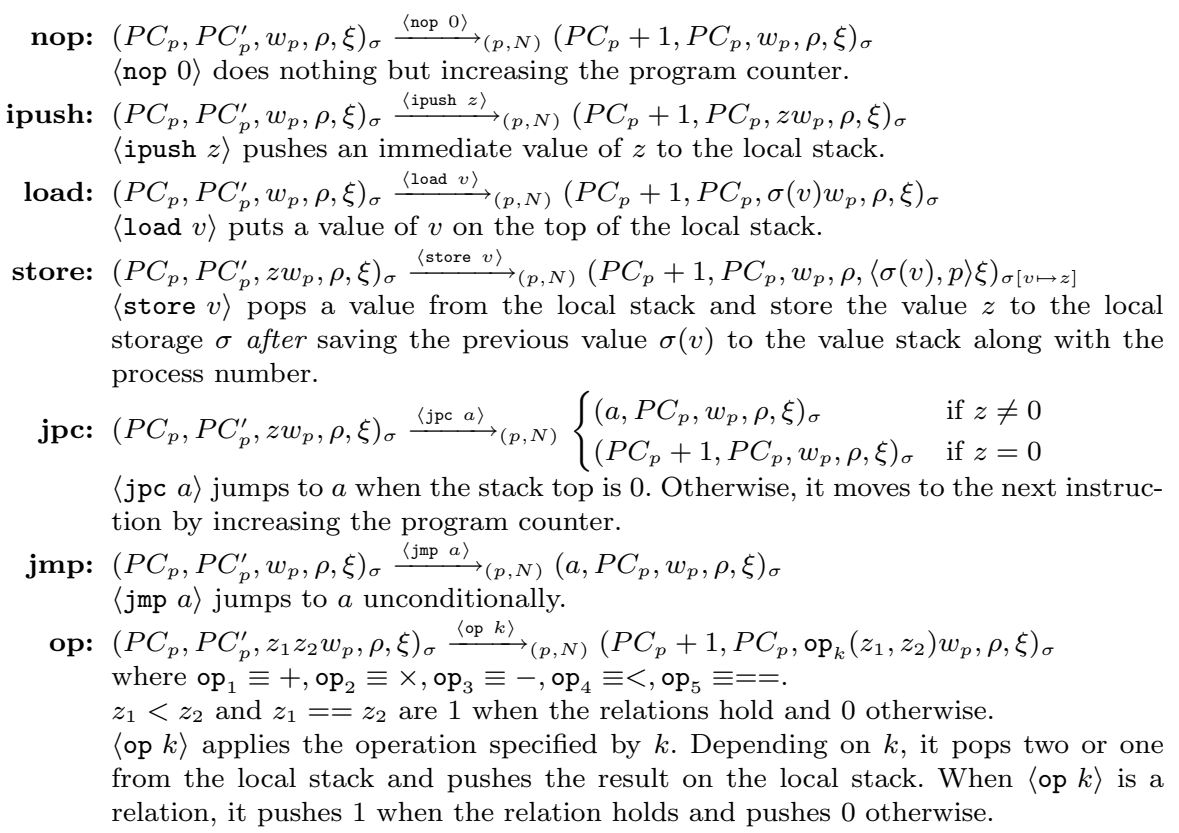

label: $\left(P C_{p}, P C_{p}^{\prime}, w_{p}, \rho, \xi\right)_{\sigma} \stackrel{\langle\text { label } n\rangle}{\longrightarrow}\left(P C_{p}+1, P C_{p}, w_{p},\left\langle n+1-P C_{p}^{\prime}, p\right\rangle \rho, \xi\right)$

$\langle$ label $n$ ) pushes the address for backward execution to the label stack where $n$ is the number of all instructions. label is the only instruction that uses $P C_{p}^{\prime}$.

rjmp: $\left(P C_{p}, P C_{p}^{\prime}, w_{p},\langle a, N+1-i\rangle \rho, \xi\right)_{\sigma} \stackrel{\langle\text { rjmp } 0\rangle}{\longrightarrow}(p, N)\left(a, P C_{p}, w_{p}, \rho, \xi\right)_{\sigma}$

$\langle$ rjmp 0$\rangle$ is a reverse jump that pops an address and a stack machine number from the label stack and jump back to the address on that process with the number.

restore: $\left(P C_{p}, P C_{p}^{\prime}, w_{p}, \rho,\langle z, N+1-i\rangle \xi\right)_{\sigma} \stackrel{\langle\text { restore } v\rangle}{\longrightarrow}(p, N)\left(P C_{p}+1, P C_{p}, z w_{p}, \rho, \xi\right)_{\sigma[v \mapsto z]}$ $\langle$ restore $v\rangle$ pops the value of $v$ and the stack machine number from the value stack on the specified stack machine.

alloc: $\left(P C_{p}, P C_{p}^{\prime}, w_{p}, \rho, \xi\right)_{\sigma} \stackrel{\langle\operatorname{arloc} v\rangle}{\longrightarrow}(p, N)\left(P C_{p}+1, P C_{p}, w_{p}, \rho, \xi\right)_{\sigma[v \mapsto 0]}$ $\langle$ alloc $v$ 〉 adds $v$ to the environment $\sigma$ and initialises $v$.

free: $\left(P C_{p}, P C_{p}^{\prime}, w_{p}, \rho, \xi\right)_{\sigma} \stackrel{\langle\text { free } v\rangle}{\longrightarrow}(p, N)\left(P C_{p}+1, P C_{p}, w_{p}, \rho, \xi\right)_{\sigma \backslash v}$ $\langle$ free $v\rangle$ removes $v$ from the environment $\sigma$. 


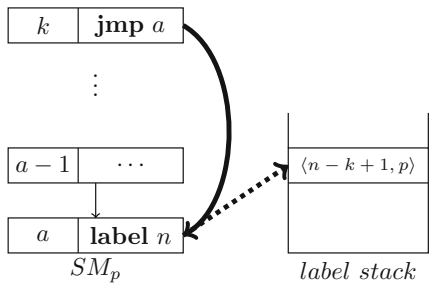

(a) Forward jump

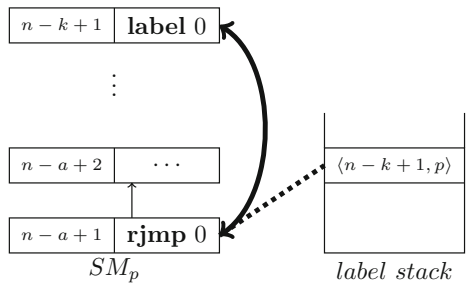

(b) Backward jump

Fig. 1. Reversing jump instructions

store and label collect the information in a forward execution. store updates $\sigma$ and $\xi$. In $\xi$, it records the value is stored by $p$. But in the backward execution, the process number is also reversed, and it stores $N+1-p$ as the backward process number. label records from which address the control reach this place.

rjmp and restore restore the information for a backward execution. rjmp corresponds to label and pops the location from $\rho$ and jump back to the location where the forward execution came from. And restore puts back the previous value from the value stack. In both cases, the stack machine must be identified where the SMid is inverted since the order of the parallel blocks is reversed ${ }^{1}$.

Figure 1 shows the mechanism of label and rjmp. label is a destination of jpc and jmp. If label is executed, it pushes the source address of that jump to the label stack. In the backward execution, label is substituted by rjmp. By popping the label stack, one of the stack machines executes rjmp and jumps back to the source address.

alloc allocates a variable slot on the stack and updates the symbol table. free removes a variable slot. $\sigma \backslash v$ removes $v$ from the domain of $\sigma$. In current target codes, alloc and free are executed only by the initial stack machine with id 0 .

\subsection{Inverting Stack Machine Code}

We do not present the detailed translation from a source program to the stack machine code here. The translator is implemented using Javacc. In the translation, label is inserted at a target of jmp and jpc. The argument of label is the length of the generated code. Since this is not known until the whole translation is done, it can be specified by back-patching. par 0 and par 1 are inserted for a parallel block.

From a forward stack machine code $s$, the backward code $i(s)$ is obtained:

$$
i(s)= \begin{cases}\varepsilon & s=\varepsilon \\ i\left(s^{\prime}\right) \operatorname{inv}(c) & s=c s^{\prime}\end{cases}
$$

\footnotetext{
${ }^{1}$ Since an address of command is uniquely assigned to a unique stack machine, it is not essential to record $p$ in $\rho$. Without $p$ in $\rho$, another table is necessary.
} 
where $\operatorname{inv}_{n}$ for each command is defined as below where $n$ is the :

$$
\begin{aligned}
\operatorname{inv}(\langle\text { store } v\rangle) & =\langle\text { restore } v\rangle, & \operatorname{inv}(\langle\operatorname{jpc} a\rangle) & =\langle\text { label } 0\rangle, \\
\operatorname{inv}(\langle\operatorname{jmp} a\rangle) & =\langle\text { label } 0\rangle, & \operatorname{inv}(\langle\text { label } n\rangle) & =\langle\operatorname{rjmp} 0\rangle, \\
\operatorname{inv}(\langle\operatorname{par} 0\rangle) & =\langle\operatorname{par} 1\rangle, & \operatorname{inv}(\langle\operatorname{par} 1\rangle) & =\langle\operatorname{par} 0\rangle, \\
\operatorname{inv}(\langle\operatorname{alloc} v\rangle) & =\langle\text { free } v\rangle, & \operatorname{inv}(\langle\text { free } v\rangle) & =\langle\operatorname{alloc} v\rangle
\end{aligned}
$$

For other command $c \operatorname{inv}(\langle c n\rangle)=\langle$ nop 0$\rangle$.

\subsection{Execution from the Initial Stack Machine}

Let $s$ be the forward stack machine code. From the construction of a program, $s$ is partitioned to:

$$
s_{I} s_{0}\langle\operatorname{par} 0\rangle s_{1}\langle\operatorname{par} 1\rangle \cdots\langle\operatorname{par} 0\rangle s_{N}\langle\operatorname{par} 1\rangle s_{F}
$$

where $M$ is the length of the code and $S M_{(0, N)}$ executes $s_{I}, s_{0}$, and $s_{F}$ where $s_{I}$ and $s_{F}$ are alloc and free for variables. $s_{0}$ is the sequential code for $S M_{0}$ to initiase the variables followed by the parallel composition. $S M_{(p, N)}$ executes $s_{p}$ in parallel for $1 \leq p \leq N$.

Figure 2 shows the overview of executing a program. The code starts on the initial stack machine $S M_{0}$. After reaching the parallel composition starting with par 0 , the $N$ stack machines run in parallel. When all the executions of parallel processes terminate, it passes the control to the initial stack machine, freeing the variable at the end. The environment $\sigma$, the label stack $\rho$, and the value stack $\xi$ are shared by all stack machines.

Let $\rho$ and $\xi$ be the label stack and the value stack. $\left\langle\operatorname{Exec}_{s}\left(P C, P C^{\prime}, w\right), \rho, \xi\right\rangle$ is a configuration of code $s$ with the initial SM. For the forward execution, the initial configuration is $\left\langle\operatorname{Exec}_{s}(1,0, \varepsilon), \varepsilon, \varepsilon\right\rangle$ and the final configuration is $\left\langle\operatorname{Exec}_{s}(M+1, M, \varepsilon), \rho_{F}, \xi_{F}\right\rangle$. The corresponding backward execution starts with $\left\langle\operatorname{Exec}_{i(s)}(1,0, \varepsilon), \rho_{F}, \xi_{F}\right\rangle$ and ends with $\left\langle\operatorname{Exec}_{i(s)}(M+1, M, \varepsilon), \varepsilon, \varepsilon\right\rangle$. While the parallel blocks are executed, the configuration is in the form:

$$
\left\langle\operatorname{Exec}_{s}^{1}\left(P C_{1}, P C_{1}^{\prime}, w_{1}\right)\|\cdots\| \operatorname{Exec}_{s}^{N}\left(P C_{N}, P C_{N}^{\prime}, \varepsilon\right), \sigma, \rho, \xi\right\rangle
$$

We define the execution of $s$ as the transition relation between configurations shown in Fig. 3. In the rules above, $P C \in s$ denotes that $P C$ points a code in $s . s(P C)$ is the code pointed by $P C$ and $\operatorname{loc}\left(s_{i}\right)$ is the address of $s_{i}$ in the stack machine code.

- Init defines the behaviour before and after the parallel composition. $\ell$ is the number of variables. $S M_{0}$ constructs the symbol table by $s_{I}$ and executes the initial sequential code $s_{0}$.

- Fork dispatches the development of the parallel blocks once it reaches the first par 0 . The program counter of stack machine $S M_{i}$ is set to $\operatorname{loc}\left(s_{i}\right)$.

- Par defines the interleaving behaviour of the parallel composition.

- Merge goes back to the initial stack machine and sets the PC to $\operatorname{loc}\left(s_{F}\right)$ once all $S M_{p}$ reaches par 0 . The execution continues with Init. 


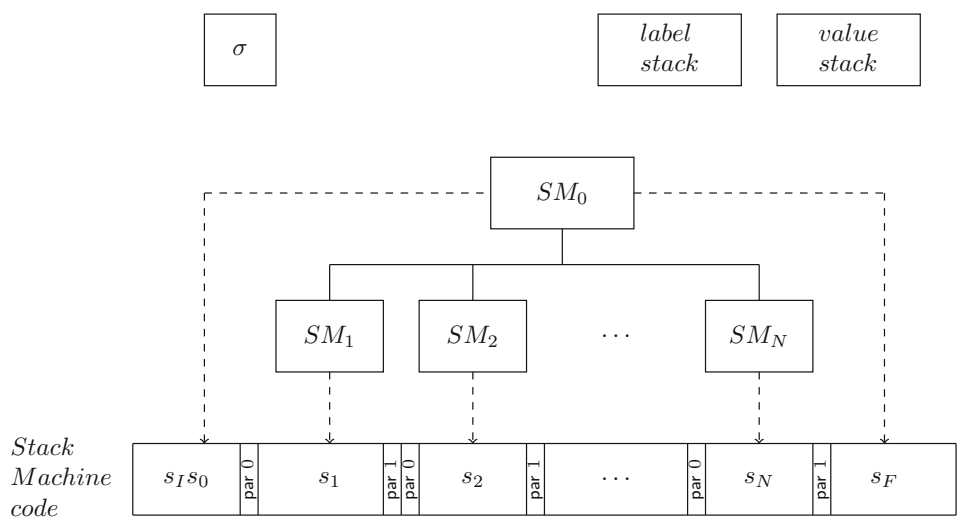

Fig. 2. Execution by stack machines

$$
\begin{aligned}
& \frac{P C^{1} \in s_{0}, s_{I}, s_{F},\left(P C^{1}, P C^{1}, w^{1}, \rho^{1}, \xi^{1}\right)_{\sigma^{1}} \stackrel{s\left(P C^{1}\right)}{\longrightarrow}(0, N)\left(P C^{2}, P C^{\prime 2}, \rho^{2}, \xi^{2}\right)_{\sigma^{2}}}{\left\langle\operatorname{Exec}_{s}\left(P C^{1}, P C^{\prime 1}, w^{1}\right), \rho^{1}, \xi^{1}\right\rangle \rightarrow\left\langle\operatorname{Exec}_{s}\left(P C^{2}, P C^{\prime 2}, w^{2}\right), \rho^{2}, \xi^{2}\right\rangle} \text { [Init] } \\
& \frac{s\left(P C_{0}\right)=\langle\operatorname{par} 0\rangle}{\left\langle\operatorname{Exec}_{s}\left(P C_{0}, P C_{0}^{\prime}, w_{0}\right), \rho_{0}, \xi_{0}\right\rangle \rightarrow} \text { [Fork] } \\
& \left\langle\operatorname{Exec}_{s}^{1}\left(\operatorname{loc}\left(s_{1}\right), P C_{0}, \varepsilon\right)\|\cdots\| \operatorname{Exec}_{s}^{N}\left(l o c\left(s_{N}\right), P C_{0}, \varepsilon\right), \sigma, \rho_{0}, \xi_{0}\right\rangle \\
& \frac{\left(P C_{p}^{1}, P C_{p}^{\prime 1}, w_{p}, \rho, \xi\right)_{\sigma} \stackrel{s\left(P C_{p}^{1}\right)}{\longrightarrow}(p, N)}{\left.\left\langle\operatorname{Exec}_{s}^{1}\left(P C_{1}, P C_{1}^{\prime}, w_{1}\right)\|\cdots\| \operatorname{Exec}_{s}^{p}\left(P C_{p}^{1}, P C_{p}^{\prime 1}, w_{p}\right)\|\cdots\| C_{p}^{\prime 2}, w_{p}^{\prime}, \rho^{\prime}, \xi^{\prime}\right)_{\sigma^{\prime}}, P C_{p}^{1} \in s_{p}\left(P C_{N}, P C_{N}^{\prime}, w_{N}\right), \sigma, \rho, \xi\right\rangle}[\mathrm{Par}] \\
& \rightarrow\left\langle\operatorname{Exec}_{s}^{1}\left(P C_{1}, P C_{1}^{\prime}, w_{1}\right)\|\cdots\| \operatorname{Exec}_{s}^{p}\left(P C_{p}^{2}, P C_{p}^{\prime 2}, w_{p}^{\prime}\right)\|\cdots\| \operatorname{Exec}_{s}^{N}\left(P C_{N}, P C_{N}^{\prime}, w_{N}^{\prime}\right), \sigma^{\prime}, \rho^{\prime}, \xi^{\prime}\right\rangle \\
& \frac{\wedge_{p} s\left(P C_{p}\right)=\langle\operatorname{par} 1\rangle}{\left\langle\operatorname{Exec}_{s}^{1}\left(P C_{1}, P C_{1}^{\prime}, w_{1}\right)\|\cdots\| \operatorname{Exec}_{s}^{N}\left(P C_{N}, P C_{N}^{\prime}, w_{N}\right), \sigma, \rho, \xi\right\rangle \rightarrow\left\langle\operatorname{Exec}_{s}\left(\operatorname{loc}\left(s_{F}\right), 0, \varepsilon\right), \sigma, \rho, \xi^{\prime}\right\rangle}[\text { Merge] }
\end{aligned}
$$

Fig. 3. Execution for code $s$

In order to implement the operational semantics, it is necessary to scan the whole stack machine code before executing the code to identify $N, \operatorname{loc}\left(s_{i}\right)$ and $\operatorname{loc}\left(s_{F}\right)$.

\section{Concluding Remarks}

We present a reversible runtime environment for simple parallel programs and its experimental implementation by Python. The reversibility mechanism is statesaving and the environment performs the back-track reversibility. The runtime environment is a set of reversible stack machines. The stack machines that execute the parallel blocks share the stacks for value-updates and jumps. Since we focus on the reversibility of states, we do not precisely reverse the forward computation. We replace the commands for computing values with nop in the 
backward code. This eases the concurrency control in the backward execution since it has no effect for states. We regard this is enough for behavioural analysis such as debugging. The approach of forward and backward executions is fundamentally similar to that of [2]. Our approach is finer-grained than [2]. This eases the implementation with the existing runtime since the runtime is often less controlled. As the result, in our approach a backward execution does not precisely undo the forward execution at the level of stack machine code. By sharing the variable environment, the label stack, and the value stack, we manage the consistency of variable updates among the stack machines running in parallel.

As related work, our stack machine is close to the basic architecture of [1] in jumping mechanism although only a sequential execution is considered. The label stack maintains the control of jumps across the parallel composition. [5] presents the reversible semantics in the functional programming style at the abstract machine level with communications and concurrency. [5] gives the operational semantics for backward execution, while our approach translates the abstract machine instructions for backwards within the single operational semantics. Our language has no built-in communication mechanism.

For future work, we need to prove the correctness of our translation by strictly formalising the behaviour of the concurrent execution of a program. The programming language in Sect. 2 limits the class of programs although the stack machine operations have more capability. Adding the nested structure of blocks and procedure is possible by extending the reference mechanism for variables. Adding recursion with the parallel composition make the number of parallel processes dynamic. We need to extend the numbering scheme for identifying the sequential processes executed in parallel and how to choose the next available process in the backward execution.

Acknowledgement. The authors thank Dr. I. Ulidowski and Dr. J. Hoey for the valuable suggestions and discussion. This work was supported by JSPS KAKENHI Grant Numbers JP17H01722 and JP17K19969.

\section{A Runtime Environment by Python}

The concrete examples and our implementation by Python are shown at https:// github.com/syuen1/RevRunTimeEnv.

A source program is compiled to the forward stack machine code.

\% java Parser "source program"

The forward stack machine code is stored in code.txt. To run the code forward,

\section{\% Python vm.py code.txt $f \mathrm{v}$}

Then, we get stack.txt,rstack.txt, and lstack.txt as the stack for variable values and the stack for labels ${ }^{2}$

\footnotetext{
${ }^{2}$ The last $\mathrm{v}$ shows the verbose mode to show all the steps. No intermediate result is shown when $\mathrm{q}$ is specified.
} 
To invert the forward code,

$\%$ Python inv.py code.txt invcode.txt

And run the backward code,

$\%$ Python vm.py invcode.txt $\mathrm{b} v$

In the backward, vm. py reads the stack files. The result for the airline ticket example is shown in the appendix.

\section{A.1 Controlling Parallel Blocks}

The runtime can be executed step-by-step choosing which parallel block is executed in the next step in both directions. The execution of the parallel blocks is controlled by the process that is running the initial stack machine. By entering the process number, the program executes one step in the forward and backward execution showing the stacks.

\section{References}

1. Axelsen, H.B., Glück, R., Yokoyama, T.: Reversible machine code and its abstract processor architecture. In: Diekert, V., Volkov, M.V., Voronkov, A. (eds.) CSR 2007. LNCS, vol. 4649, pp. 56-69. Springer, Heidelberg (2007). https://doi.org/10.1007/ 978-3-540-74510-5_9

2. Hoey, J., Ulidowski, I.: Reversible imperative parallel programs and debugging. In: Thomsen, M.K., Soeken, M. (eds.) RC 2019. LNCS, vol. 11497, pp. 108-127. Springer, Cham (2019). https://doi.org/10.1007/978-3-030-21500-2_7

3. Hoey, J., Ulidowski, I., Yuen, S.: Reversing parallel programs with blocks and procedures. In: EXPRESS/SOS 2018, Beijing, China, 3 September 2018, EPTCS, vol. 276, pp. 69-86 (2018)

4. Levin, R.Y., Sherman, A.T.: A note on Bennett's time-space tradeoff for reversible computation. SIAM J. Comput. 19(4), 673-677 (1990)

5. Lienhardt, M., Lanese, I., Mezzina, C.A., Stefani, J.-B.: A reversible abstract machine and its space overhead. In: Giese, H., Rosu, G. (eds.) FMOODS/FORTE -2012. LNCS, vol. 7273, pp. 1-17. Springer, Heidelberg (2012). https://doi.org/10. 1007/978-3-642-30793-5_1

6. Thomsen, M.K., Axelsen, H.B.: Interpretation and programming of the reversible functional language RFUN. In: IFL 2015, Koblenz, Germany, 14-16 September 2015, pp. 8:1-8:13. ACM (2015)

7. Yokoyama, T., Glück, R.: A reversible programming language and its invertible self-interpreter. In: PEPM 2007, pp. 144-153. ACM (2007) 